O'Kelly, M., Anisimov, V., Campbell, C. and Hamilton, S. (2017) Proposed best practice for projects that involve modelling and simulation. Pharmaceutical Statistics, 16(2), pp. 107-113. (doi: 10.1002/pst.1789)

There may be differences between this version and the published version. You are advised to consult the publisher's version if you wish to cite from it.

This is the peer-reviewed version of the following article: O'Kelly, M., Anisimov, V., Campbell, C. and Hamilton, S. (2017) Proposed best practice for projects that involve modelling and simulation. Pharmaceutical Statistics, 16(2), pp. 107-113, which has been published in final form at 10.1002/pst.1789. This article may be used for non-commercial purposes in accordance with Wiley Terms and Conditions for Self-Archiving.

http://eprints.gla.ac.uk/137913/

Deposited on: 07 March 2017

Enlighten - Research publications by members of the University of Glasgow http://eprints.gla.ac.uk 


\title{
Proposed Best Practice for Projects that Involve Modelling and Simulation
}

\author{
Authors \\ O’Kelly $\mathrm{M}^{1}$, Anisimov $\mathrm{V}^{2}$, Campbell $\mathrm{C}^{3}$, Hamilton $\mathrm{S}^{1}$. \\ ${ }^{1}$ Quintiles (Ireland). \\ ${ }^{2}$ University of Glasgow \\ ${ }^{3}$ Mango Solutions (UK)
}

\begin{abstract}
Modelling and simulation has been used in many ways when developing new treatments. To be useful and credible, it is generally agreed that modelling and simulation should be undertaken according to some kind of Best Practice. A number of authors have suggested elements required for Best Practice in modelling and simulation. Elements that have been suggested include the pre-specification of goals, assumptions, methods and outputs.

However, a project that involves modelling and simulation could be simple or complex; and could be of relatively low or high importance to the project. It has been argued that the level of detail and the strictness of pre-specification should be allowed to vary, depending on the complexity and importance of the project. This Best Practice document describes the elements required for the specification of a project, and requires that the practitioner justify in the specification the omission of any of the elements and, in addition, justify the level of detail provided about each element. This document is an initiative of the Special Interest Group (SIG) for Modelling and Simulation. The SIG for Modelling and Simulation is a body open to members of Statisticians in the Pharmaceutical Industry (PSI) and the European Federation of Statisticians in the Pharmaceutical Industry (EFSPI). Examples of a very detailed specification and a less detailed specification are included as appendices.

Keywords: Modelling and simulation; Best practice; Monte Carlo technique; Prespecification, Quality control.

\section{Scope of this document}

We note that any project involving simulation has analytic elements, and involves model development, model selection and model checking. For purposes of this document, a project that involves modelling and simulation is one that has the objective of answering specified scientific or business questions using a statistical model or models, with the estimates from the model having a stochastic element; and/or having combinations of attributes that are not present together in a single dataset or in the individual assumptions used by the model; and/or extrapolating beyond the directly specified assumptions and/or beyond the support provided by the data on which the model is based. This Best Practice document applies to all projects that involve modelling and simulation as defined thus. See Table I for key definitions.
\end{abstract}


Rather than attempting to prescribe the content of inputs, methods and outputs pertaining to best practice, this document enumerates the required elements of a specification, and for best practice requires that the specification justify the level of detail and stringency of each element specified.

-Table I about here-

The document does not cover the details of investigative PK/PD modelling, but is designed to be generally applicable to modelling and simulation as used in any way in the development of new clinical treatments; it addresses the need for pre-specification in model informed clinical trial design and simulation. As part of best practice this document gives points to consider when deciding whether or not to use simulation.

\section{Objective of this document}

The objective of this document is to foster best practice in projects involving modelling and simulation. The document encourages appropriate use of modelling and simulation by describing the settings where simulation may and may not be useful; then the document proposes key elements of modelling and simulation that should be specified in advance.

\subsection{The role of the specification}

The specification for a project involving modelling and simulation should be reviewed by team members, including those who will make use of the results of the project. Appropriate specification is necessary for best practice for a number of reasons. Firstly, a specification allows those who will make use of results to judge in advance whether in fact those results will help to answer the research question, and to contribute to improving the plan so as to answer the research question better. Secondly, when the project team uses an appropriate specification, the team will be enabled to act consistently to provide the planned outputs. Thirdly, if a project is adequately described in a specification, the degree to which the project achieved its specified goals can be assessed and the results reproduced. Thus appropriate specification encourages best practice in communication, planning and evaluation of the modelling-and-simulation project.

It is recognized that the level of stringency with which a project is specified, carried out and checked should take into account the importance of that project. For example, at the European Medicines Agency-European Federation of Pharmaceutical Industries and Associations (EMA-EFPIA) Modelling and Simulation Workshop in 2011 it was "agreed that different standards will apply depending on the impact of M(odelling )\&S(imulation)." [1]; and an EMA speaker noted that there should be "Different standards for different exercises (L(ow) M(edium) H(igh importance))" [2]. Projects that involve modelling and simulation thus vary widely with regard to the level of detailed pre-specification and the level of quality control that can be justified as Best Practice. While this document does not prescribe the contents of elements of the specification in detail, in some cases it provides suggestions. Appendices containing examples of specifications for projects requiring low and medium/high levels of stringency are available as separate documents.

In reality, deviations from the specification may occur while undertaking a project.

Furthermore, projects that involve modelling and simulation are often iterative. That is, a first run of the simulation and analysis often gives rise to questions that can be well answered by a re-run of the modelling and simulation, with amendments to the specification. With 
appropriate recording of deviations and amendments, the impact of these is auditable and can be assessed (see Section 3.4 "Quality Control" and Section 4 "Changes to the specification").

\section{Introduction}

Modelling and simulation uses rules derived from the observation of existing conditions in new but related conditions. Modelling and simulation allows the analyst to interrogate the interaction between one or more models and new assumptions. Modelling and simulation can be used in many ways when developing a treatment, from drug discovery to health technology assessment. In the simplest case, modelling and simulation could provide predictions from a well specified model, under a new set of conditions. At its most complex, modelling and simulation could use a variety of models, methodologies, and inferences, and/or require assumptions for situations that have not yet been observed or that can be derived only indirectly from observed data. The risk of the methods used may be commensurate with the differences vis-à-vis the previously observed conditions, and with the complexity of the modelling and simulation. The reporting of such studies should communicate this clearly.

Modelling and simulation typically involves applying model-based mathematical/statistical results that can be used as evidence in drawing conclusions about the behaviour of specific project characteristics.

A note on terminology: statistical models make use of assumptions, but model parameters tend to be estimated from real or historical data. Sometime we can use Bayesian prior parameters which can also be supported by historical information. If the project is developing over time, typically the estimates of model parameters are updated using interim data to update the posterior estimates. However, in projects involving modelling and simulation, there is in practice great flexibility in the degree to which the model is derived from a mixture of assumptions and of data. Sometimes, the values of parameters will be assumed, based loosely on historic data or on expert opinion. Therefore, this document tends to use the term "assumptions and data" where often the term "data" would be used.

\subsection{Closed-form approaches vs. simulation}

In projects involving modelling and simulation, either closed-form solutions/mathematical formulae can be used (which are known or derived for the purposes of the projects), or use may be made of Monte Carlo simulation, or the results can be obtained by using a combination of both approaches.

Typically each project involving modelling and simulation consists of several stages. In the first stage, models are defined that link the initial assumptions or input data to the outcome(s) that need to be analysed. The second part involves the process of generating outcomes which can be done either by applying closed-form solutions or by using Monte Carlo simulation. Then the results (outcomes) are analysed with the objective of testing particular hypotheses or proving the existence of specific properties. As usual this analysis is made using statistical tests based on analytic solutions, e.g. testing the equality of means.

For some projects both closed-form analytic solutions and Monte Carlo simulation may be used. Typically, closed-form solutions can be derived for projects where we use standard types of distributions and outcome measures for which formulae are known, e.g. Gaussian or Poisson models without truncation. As usual, using closed-form expressions is preferred as 
this reduces the time required for analysis and eliminates errors due to the simulation process itself ("stochastic variability"). However, for many projects it may be difficult to derive closed-form solutions. In these cases Monte Carlo simulation techniques are used, sometimes combining the results obtained by simulation using analytic techniques.

Note that for some projects it might be appropriate instead of exact distributions to use approximations - often a normal or Poisson approximation. In the case of a normal approximation, it is enough to evaluate the mean and standard deviation (SD) of the resulting outcome, where in many cases the mean and SD can be calculated directly. In such cases the project may not require Monte Carlo simulation.

Examples of a project with a model whose estimates have a stochastic element:

- A project that simulates patient outcomes in order to assess the operating characteristics of candidate analysis methods.

- A project that predicts future patient enrolment at interim time.

An example of a project with estimates that have combinations of attributes that are not present in the data or individual assumptions used by the model:

- A simulation of a dose-finding study with safety and efficacy profiles taken from separate sources for a range of doses, where both safety and efficacy must be taken into account together in assessing the risks and benefits of a design to identify viable doses of a new treatment.

An example of a model that extrapolates beyond the assumptions or data:

- A PK model that estimates serum concentrations for obese patients from those of nonobese patients.

Examples of projects that may allow closed-form solutions:

- A project which requires the evaluation of the cumulative outcome where the individual outcome is the number of events during the exposure time, where the rate of events may depend on subject covariates. In this case, if the rates and exposure times are given, the cumulative outcome has a Poisson distribution, in which case Monte Carlo simulation is not required.

- For a large number of subjects, it would be enough to evaluate the mean and SD of individual outcome. This can be done analytically only for some special cases, e.g. where the rates are known and exposure times are either known and have some specific type of distribution, e.g. uniform or gamma. In this case, it is also possible to use a normal approximation for cumulative outcome and avoid Monte Carlo simulation.

- For some projects, the opportunity to use closed-form solutions may depend on the logistics of project design. For example, for modelling global patient enrolment where there are not restrictions on the number of patients in different centres/regions we can use mixed Poisson process models that typically allow closed-form solutions [3]. However, if there are caps on the number of recruited patients in some regions, then 
analytic solutions become rather complicated and the natural way would be to use Monte Carlo simulation.

We note that in some projects the derived analytic relations and/or hypotheses about the behaviour of particular characteristics may benefit from verification by using Monte Carlo simulation of a variety of scenarios and illustration of the results by plots and tables. There are many papers (see e.g., [3]) that use Monte Carlo simulation as the engine to test and verify the analytic results.

\section{The Project Specification}

Unless otherwise stated, a specification for a project involving modelling and simulation should have headings/subheadings corresponding to each of the headings/subheadings of this section, "The Project Specification" and its subsections. Thus this section constitutes a check list that can be used when drafting a specification for a project involving modelling and simulation. The Introduction of the specification, the Presentation of results and the Summary if included, should be understandable by non-statisticians, the audience for all other sections of the specification should be the statistical team (statisticians and statistical programmers).

\subsection{Summary}

A summary may be included. If included, the summary should as a minimum state the objective of the project.

\subsection{Introduction of the specification}

The introduction of the specification should describe the objective of the modelling and simulation and state how the objective is addressed by the modelling and simulation.

To the extent required by the project, the introduction should describe the clinical and operational setup (e.g. study design, indication, visits, treatments, enrolment scenarios, expected time to complete study, as appropriate); and the metrics and criteria used to draw conclusions.

In some cases, it may be possible to provide in the Introduction a justification for a level of detail covering all elements of the specification; otherwise, justification of the level of detail provided for each element, including the Introduction, will be required.

\subsection{Simulation and analysis/design}

The description of the simulation, analysis and design will have a number of elements. The level of detail with which each element is described should be justified.

The description of the simulation should allow the reader to understand how the simulation is planned to be carried out. At its most detailed, this section should allow the reader to reproduce the simulations.

The elements of the "Simulation and analysis" section of a specification are now covered individually.

\subsubsection{Scenarios assumed and assumptions made}

To the extent required by the project, the following should be described:

- the assumptions made and their basis; 
- a justification of those assumptions;

- the scenarios under these assumptions and their clinical significance.

The level of detail with which these are described should be justified here, if this is not done elsewhere.

Scenarios should cover likely assumptions but, depending on the importance of the project, should also cover less-likely assumptions, preferably including assumptions unfavourable to the sponsor of the project, insofar as this can be planned in advance. If applicable, the degree to which assumptions are favourable and unfavourable to the sponsor should be stated and this statement justified. Where applicable, consider including a scenario where the null hypothesis pertains.

If prior distributions are assumed for parameters, these distributions should be justified.

As well as scenarios (often specified in terms of assumptions about model parameters), other assumptions of the model should be stated and justified. The specification may include a plan or recommendation for actions to verify the appropriateness of the model(s) used - see e.g. [4] for a standard text on model checking.

\subsubsection{Sensitivity analyses}

The specification should plan for, describe and justify analyses that assess the sensitivity of the conclusions to the primary assumptions of the project, and should describe the output of these analyses. This may be done in the course of specifying the variety of scenarios in Section 3.3.1 "Scenarios assumed and assumptions made". If no sensitivity analyses are performed, this omission should be justified. In particular, consider assessing sensitivity to missing data. An assessment of sensitivity to compliance may be considered.

\subsubsection{Datasets generated}

The process that generates the datasets should be described and the level of detail of this description justified. If suitable data could be simulated using bootstrap methods, it may be appropriate to provide justification for the use or non-use of bootstrap methods to generate the data sets.

\subsubsection{Statistical analysis}

The statistical analysis performed on the simulated datasets should be described and the level of detail of this description justified.

\subsubsection{Operating characteristics}

This section should describe the procedures used to calculate and justify estimated power to detect relevant differences in criteria used for conclusions. The section should also describe the assumptions made for this estimate of power and include description of the basis of those assumptions. If relevant to the objective of the project, this section should describe the procedures used to calculate type I error (probability of false positive finding for a nullhypothesis scenario). If Monte Carlo simulation is used, the number of simulations should be justified and the simulation error should be estimated. Burton et al. [5] give useful formulae to help with these calculations. It is recognized that feasibility may be a factor in deciding on the power/number of simulations.

The level of detail of the description of the operating characteristics should be justified. 


\subsubsection{Logistics}

Modelling and simulation can be affected by the parameters of the execution environment. Differences between hardware and operating systems can cause differences in absolute numeric values, which could be magnified using certain methods. The execution environment should be specified in sufficient detail to allow the results to be recreated.

The modelling and simulation will be affected by the implementation of the code. The specification should name software tools used, and the version numbers of those tools. This includes version numbers for the coding environment (R, SAS, etc.), modules of add-in code, and user generated commands.

The specification may include recommendations for optimising programming code with respect to speed, use of computer memory and/or development time

If the code is not provided as an appendix/supplementary materials, the location of the code repository should be specified.

Give details of the mechanism used for creating pseudo-random variables if this is not apparent from the description of the logistics. State the seeds to be used.

The level of detail of the description of the logistics should be justified.

If applicable, this section should describe contingency actions for foreseeable practical problems such as lack of convergence or clinically unrealistic simulated values, and should justify the level of detail used for this description.

\subsection{Quality control}

As with other elements, the level of quality control (QC) for a project involving modelling and simulation may vary depending on the importance of a project. Projects are often categorised as of low, medium or high importance. An example of a project of low importance could be a simulation that is used to verify a known formula. An example of a project of medium importance could be where conventional analyses produce valid results but simulation is used to provide additional summaries that are easier to interpret clinically no new conclusions may be drawn from the results of the simulation, but the simulation results will contribute to the decision-making process. An example of a project of high importance could be where results from modelling and simulation are used in a regulatory submission to support a use of a new treatment, for a subgroup of patients not directly assessed in a randomized clinical trial.

For projects of low importance, the specification should be reviewed independently. Software tools used for generating results may be software accepted.

For projects of medium importance, the specification should be reviewed independently. Software tools used for generating results should be software accepted. It should be verified that under known conditions the analytical results display expected characteristics. In addition, the user code may be peer reviewed, and may be unit tested where appropriate to verify expected behaviour. 
For projects of high importance, the specification should be reviewed independently.

Software tools used for generating results should be software accepted. The user code should be peer reviewed. Unit testing may be used to verify expected behaviour. The project results should be quality controlled via independent programming. Independent programming is the comparison of the outputs from two independently programmed models/simulations that follow the same specification. This can be a reliable QC of both the correctness of the computer code and the clarity of the specification, both of which are sources of error in practice.

Quality control of the generation of the data can be separated from QC of the analysis and presentation of results. The QC of the generation of the data could be performed via independent programming using a common seed and agreed outline of the order of the implementation of steps (including agreed order of sorting of data pertaining to the generation of the pseudo-random variables).

The specification may require that programs and/or datasets be auditable.

The QC strategy should be described in the specification. The specification should justify the level of planned QC.

\subsection{Presentation of results}

The specification should describe the items planned to be estimated overall. The items planned to be estimated at each simulation should also be described, or the omission of this description justified. If there is a hierarchy of summaries, this should be described and the estimates pertaining to each level of the hierarchy described, or its omission justified. It is recognized that the presentation of results may vary depending upon the audience for the presentation. This section of the specification should describe how the results will be presented and justify the contents, format and level of detail of the presentation for each planned audience. Graphical presentations should be considered.

This section should also describe how the bias, accuracy and coverage of the results will be presented, or justify the omission of these items from the presentation.

Confidence intervals should be presented for results, or their omission justified.

As appropriate, this section should describe the format for storage of the items estimated and justify the level of detail used for this description.

\section{Changes to the Specification}

The specification for a project that involves modelling and simulation should when finalised have a dated signature attached to it in an auditable fashion, or a justification for the omission of this should be given in the specification. The date associated with the first final version of the specification should at the latest be prior to the date of the first generation of simulated data used to provide project results. If the date is later, a justification of this should be included.

As noted (section 1, "Scope of this document"), projects involving modelling and simulation tend to be iterative, and thus amendments to the specification are often necessary. At least for projects of medium and high importance, amendments to the specification made after the first signed dated final version should be clearly indicated as amendments. Such amendments 
should be signed, dated and justified, or the omission of these particulars should itself be justified. The version number should reflect amendments. Among other ways of recording amendments the following may be considered:

- revision history

- formal amendment, giving reason for change (analogous to protocol amendment)

- including changes or new versions of the specification as appendices.

Programs and/or datasets may be auditable (see Section 3.4 "Quality Control").

Project programs and/or outputs should be linked in an auditable manner to the version of the specification used, or the lack of such links should be justified in the section of the specification that specifies Quality Control.

\section{Acknowledgements}

This document is an initiative of the Special Interest Group (SIG) for Modelling and Simulation. The SIG for Modelling and Simulation is a body open to members of Statisticians in the Pharmaceutical Industry (PSI) and the European Federation of Statisticians in the Pharmaceutical Industry (EFSPI). The authors thank Jason Wang and other PSI members for their useful feedback on drafts of this document, with special thanks to Chris Jennison for his important contribution to the Modelling and Simulation SIG Hackathon (February 2015, London), an exercise involving about 15 PSI members that helped to improve the Best Practice document greatly. We also thank those who attended the Hackathon. A number of valuable papers have been published on best practice for projects that involve modelling and simulation. This document benefits from work by, among others, Bonate et al. [6], US Food and Drug Administration [7], Burton et al. [5], Gaydos et al. [8], Smith et al. [9] and Burman et al. [10]. See also Marshall et al. [11] on the work of EFPIA on Good Practices in Model Informed Drug Discovery and Development (MID3).

The authors contributed to this paper as follows: Michael O'Kelly created the draft document; Vladimir Anisimov added Section 3.1 on closed form approaches vs. simulation; Chris Campbell added much of Sections 4.3.5, 4.4 and 5 on Logistics, Quality Control and Changes to the Specification. Sinéad Hamilton added the sample specification in the Appendix, entitled "Using simulated data to assess analyses of negative binomial outcomes with missing data". All authors reviewed the final draft. Vladimir Anisimov was affiliated to Quintiles (UK) when he contributed to the paper.

7. References

1. European Medicines Agency. EFPIA-EMA Modelling and Simulation Workshop Report. EMA-EFPIA Modelling and Simulation Workshop, 30 Nov - 1 Dec 2011, London. Available at http://www.ema.europa.eu/docs/en_GB/document_library/Report/2012/05/WC50012 7118.pdf. Accessed 31 March 2016.

2. Hemmings R. $M \& S$ good practices and next steps. EMA-EFPIA Modelling and Simulation Workshop, 30 Nov - 1 Dec 2011, London. Available at 
https://www.youtube.com/watch? $\mathrm{v}=\mathrm{BSsaUmMuUAE \& index}=12 \&$ list=PL7K5 dNgKn awY96v4FlgjTwUFAgUCXrRmw. Accessed 31 March 2016.

3. Anisimov VV, Fedorov VV. Modelling, prediction and adaptive adjustment of recruitment in multicentre trials. Statistics in Medicine 2007; 26: 4958-4975.

4. Cook R, Weisberg S. Applied regression including computing and graphics. Chichester: John Wiley and Sons, 1999.

5. Burton A, Altman D, Royston P, Holder R. The design of simulation studies in medical statistics. Statistics in medicine 2006; 25: 4279-4292.

6. Bonate P, Gillespie W, Ludden T, Rubin DB, Stanski D. Simulation in drug development: good practices. In Draft publication of the Centre for Drug Development Science (CDDS), Holford NHG, Hale M, Ko H, Steimer J-L, Sheiner LB, Peck CC (eds). University of California: San Francisco; 1999; pp. 1-18.

7. US Food and Drug Administration, Center for Drug Evaluation and Research, Center for Biologics Evaluation and Research. Guidance for industry: population pharmacokinetics. US Food and Drug Administration: Rockville, MD, 1999.

8. Gaydos B, Anderson K, Berry D, Burnham N, Chuang-Stein C, Dudinak J, Fardipoor P, Gallo P, Givens S, Lowis R, Maca J, Pinheiro J, Pritchett Y, Krams M. Good practices for adaptive clinical trials. Drug Information Journal 2009; 43: 539-556.

9. Smith M, Marshall A. Importance of protocols for simulation studies in clinical drug development. Statistical Methods in Medical Research 2010; 20: 613-622.

10. Burman C-F, Wiklund S. Modelling and simulation in the pharmaceutical industrysome reflections. Pharmaceutical Statistics 2011; 10: 508-516.

11. Marshall S, Burghaus R, Cosson V, Cheung S, Chenel M, DellaPasqua O, Frey N, Hamren B, Harnisch L, Ivanow F, Kerbusch T, Lippert J, Milligan P, Rohou S, Staab A, Steimer J, Tornøe C, Visser S. Good Practices in Model-Informed Drug Discovery and Development (MID3): practice, application and documentation. CPT: Pharmacometrics \& Systems Pharmacology 2016; Accepted Article, doi: 10.1002/psp4.12049 


\begin{tabular}{|c|c|}
\hline Term & Definition \\
\hline $\begin{array}{l}\text { Modelling and } \\
\text { simulation } \\
\text { project }\end{array}$ & $\begin{array}{l}\text { Project to answer specified scientific and business questions using } \\
\text { model(s) with estimates that: } \\
\text { - have a stochastic element, and/or } \\
\text { - have combinations of attributes that are not present together in a } \\
\text { single dataset or in the individual assumptions used by the model, } \\
\text { and/or } \\
\text { - extrapolate beyond the directly specified assumptions and/or } \\
\text { beyond the support provided by the data used by the model. }\end{array}$ \\
\hline $\begin{array}{l}\text { Monte Carlo } \\
\text { simulation }\end{array}$ & $\begin{array}{l}\text { Simulation that uses repeated random sampling to obtain results; the } \\
\text { random sampling may be pseudo-random, implemented via a computer }\end{array}$ \\
\hline $\begin{array}{l}\text { Project } \\
\text { importance }\end{array}$ & $\begin{array}{l}\text { - the importance of the results of the project for internal decision } \\
\text { making, } \\
\text { the importance of the contribution to regulatory approval of the } \\
\text { evidence resulting from the project, } \\
\text { more generally, the scientific importance envisaged with regard to } \\
\text { the results of the project. } \\
\text { See Section } 3.4 \text { "Quality Control" for examples of levels of } \\
\text { importance. }\end{array}$ \\
\hline $\begin{array}{l}\text { Software } \\
\text { acceptance }\end{array}$ & $\begin{array}{l}\text { The process of defining the scope of a software tool, and checking that } \\
\text { the software meets that specification, with an appropriate thoroughness } \\
\text { based on project importance: } \\
\text { - ad hoc testing (medium) } \\
\text { - software specification, manufacturer's installation } \\
\text { qualification/operational qualification/performance qualification } \\
\text { (IQOQPQ) procedure, and formal acceptance testing (high) }\end{array}$ \\
\hline Stochastic & $\begin{array}{l}\text { Having an inherently random element, and therefore not capable of } \\
\text { having its value predicted exactly }\end{array}$ \\
\hline Unit testing & $\begin{array}{l}\text { Testing individual units of source code to determine whether they are } \\
\text { fit for use }\end{array}$ \\
\hline
\end{tabular}

